\title{
Criterios de selección de mano de obra calificada para un proyecto de construcción de redes de alcantarillado y agua potable en la ciudad de Cuenca
}

Criteria for selecting qualified labor for a construction project of sewerage and drinking water networks in the city of Cuenca

Nelson Santiago Urgilés Martínez. ${ }^{1}$ \& Juan-Carlos Ortega-Castro. ${ }^{2}$

\begin{abstract}
.
DOI: https://doi.org/10.33262/concienciadigital.v4i3.1763

Introduction: In order for civil constructions to be completed within the specified deadlines and not incur high costs due to delays, it is necessary to have a qualified workforce that is capable of executing the construction actions with high quality standards in the established times. It is for this reason that it is necessary to identify the necessary characteristics that applicants must possess before assembling the workforce. Objective: Determine selection criteria for qualified labor for civil constructions focused on drinking water and sewerage networks. Methodology: To meet the objective, a bibliographic compilation on selection criteria and factors that affect the productivity of labor in civil projects began. With this information, we proceeded to develop a data collection tool to apply them to a population of civil engineers in the city of Cuenca to inquire about the characteristics that, in their perception, they consider important to consider a worker as qualified. Results: Among the most relevant results, it was determined that the factors of the workforce such as physical condition, age, health and build are not the only determinants to qualify the workers. Conclusion: With all the above raised, it was concluded that the empirical knowledge and skills that the workforce can offer has the same relevance as physical condition and health.
\end{abstract}

\footnotetext{
${ }^{1}$ Universidad Católica de Cuenca, Facultad de Arquitectura. Cuenca, Ecuador, nelson.urgiles1@est.ucacue.edu.ec ORCID https://orcid.org/0000-0003-1583-0448

${ }^{2}$ Universidad Católica de Cuenca, Facultad de Arquitectura. Cuenca, Ecuador, jcortegac@ucacue.edu.ec ORCID https://orcid.org/0000-0001-6496-4325
} 
Keywords: criteria, selection, labor, construction, civil

\section{Resumen.}

Introducción: Para que las construcciones civiles se cumplan en los plazos determinados y no incurran en costos elevados por retrasos, es necesario contar con mano de obra calificada que sea capaz de ejecutar las acciones constructivas con altos estándares de calidad en los tiempos planteados. Es por esta razón, que es necesario identificar las características necesarias que deben poseer los aspirantes antes de armar la plantilla de trabajo. Objetivo: Determinar criterios de selección de mano de obra calificada para construcciones civiles enfocadas en redes de agua potable y alcantarillado. Metodología: Para cumplir con el objetivo, se inició con una recopilación bibliográfica sobre criterios de selección y factores que afectan la productividad de la mano de obra en los proyectos civiles. Con esta información se procedió a elaborar una herramienta de recolección de datos para aplicarlos a una población de ingenieros civiles en la ciudad de Cuenca para indagar sobre cuáles son las características que, bajo su percepción, consideran importantes para considerar a un obrero como calificado. Resultados: Entre los resultados con mayor relevancia se determinó que los factores propios de la mano de obra como estado físico, edad, salud y contextura no son los únicos determinantes para calificar a los obreros. Conclusión: Con todo lo anterior planteado se llegó a la conclusión que los conocimientos empíricos y habilidades que puede ofrecer la mano de obra tiene la misma relevancia que el estado físico y la salud.

Palabras claves: criterios, selección, mano de obra, construcció, civil

\section{Introducción.}

Durante generaciones, las construcciones civiles formaron parte de la identidad de los pueblos, pues son un claro indicativo de una prospera actividad económica, representan elementos socio - culturales propios de una región y satisfacen necesidades vitales para el normal desarrollo de las actividades socio - económicas de las sociedades (Cruz \& Centeno, 2019). A lo anterior descrito, Giordani \& Leone (2015) en su investigación, conceptualizan la construcción civil como un conjunto de obras en la que interviene un grupo multidisciplinario de profesionales del área encargados de supervisar, planificar, dirigir y ejecutar las acciones de construcción en beneficio de los usuarios en un espacio de instalación que puede ser de carácter público o privado.

Siguiendo con el pensamiento anterior, en los procesos de construcción civil intervienen de manera directa e indirecta, un número indeterminado de personas e instituciones que pueden afectar en la calidad de los resultados finales, es por esta razón, que cada proyecto a realizarse debe tener una adecuada planificación de recursos, tanto humanos como materiales. La planificación de una obra civil se puede definir como:

El proceso de definir, coordinar y determinar el orden en que deben realizarse las actividades con el fin de lograr la más eficiente y económica utilización de los equipos, elementos y recursos de que se dispone y de eliminar 
diversificaciones innecesarias de los esfuerzos, proceso que se establece o define en un plan de trabajo, el cual debe ser controlado a lo largo de la faena para saber si se está cumpliendo o si debe ser sometido a una revisión o modificación a fin de que se pueda cumplir con el objetivo final fijado. (Universidad de Chile [CEC], 2003, pág. 1)

Como se refirió en el apartado anterior, las operaciones de construcción civil están condicionados por la planificación de las obras, puesto que, desde la gestión, se puede asegurar la entrega oportuna de los proyectos en los plazos fijados y garantizar estándares de calidad a bajo costo de producción. Pese a lo mencionado, estos objetivos no siempre se cumplen, pues, como manifiesta Morocho (2015): "La construcción civil es considerada como una actividad de baja productividad por la imprecisión en cuanto a costos, plazos y calidad (...) el principal problema encontrado es la deficiencia de los procesos constructivos por la mano de obra no calificada" (p.126). El problema anterior descrito, es una causa común en cualquier proceso en la cual, la calidad y eficiencia depende directa e indirectamente del factor humano, ya que, la efectividad de las acciones de las personas está condicionado a varios factores como; psicológicos, físicos, educativos, medioambientales, sociales y familiares que influyen en su proceder y puede repercutir en el resultado final de las obras civiles (Salanova, Gracia, \& Lorente, 2007)

A lo expuesto, la mano de obra es considerada como un recurso activo dentro de la gestión de procesos constructivos que influye directamente en los tiempos de ejecución de las obras y, por lo tanto, también interviene en el costo total del mismo, o dicho de otro modo es un factor que incide en el abaratamiento o encarecimiento de las construcciones (Padilla, 2016). Según estadísticas presentadas por la revista Mundo Constructor (2021) la influencia de la mano de obra representa del $30 \%$ al $35 \%$ del costo total de un proyecto en condiciones normales.

Por lo mencionado, no es sorpresa que investigaciones como la de los autores Mejía \& Hernández (2007) mencionen que el rendimiento de la mano de obra en la ejecución de los procesos de construcción es inversamente proporcional a la calidad de los recursos humanos utilizados, es por esta razón, que varios estudios, se enfocan en determinar los factores que afectan la productividad de la mano de obra, para realizar contrataciones certeras. Uno de los autores más relevantes en este campo es Botero (2002) el cual menciona que:

La mano de obra, como uno de los componentes en el proceso productivo, aparece como una de las variables que afectan la productividad. Como uno de los objetivos de todas las empresas es ser más competitivos, mejorando la productividad de sus procesos productivos, se hace necesario conocer los diferentes factores que afectan la mano de obra, clasificándolos y determinando una metodología para medir su afectación en los rendimientos y consumos de mano de obra de los diferentes procesos de producción. (p.11)

En este sentido, como se muestra en la tabla 1, el autor menciona siete factores que afectan el rendimiento de la mano de obra en las construcciones civiles. 
Tabla 1. Factores que afectan el rendimiento de la mano de obra

\begin{tabular}{|c|c|}
\hline Factor & Descripción \\
\hline $\begin{array}{l}\text { Economía } \\
\text { general }\end{array}$ & $\begin{array}{l}\text { Hace referencia al estado económico de la región en la que se } \\
\text { desenvuelve la obra en la cuales se deben considerar las tendencias } \\
\text { de los negocios de la zona, la proporción del proyecto y las } \\
\text { condiciones de empleo. }\end{array}$ \\
\hline $\begin{array}{l}\text { Aspectos } \\
\text { laborales }\end{array}$ & $\begin{array}{l}\text { Este factor influye en la interacción entre las condiciones laborales } \\
\text { que ofrece el proyecto y la productividad del obrero pues interviene } \\
\text { variables como; el tipo de contrato, los procesos de sindicalización, } \\
\text { los incentivos económicos por metas cumplidas, la coherencia entre } \\
\text { los salarios con las labores requeridas y el ambiente de trabajo en } \\
\text { general. }\end{array}$ \\
\hline Clima & $\begin{array}{l}\text { Este apartado identifica las condiciones medioambientales que } \\
\text { interfieren con la productividad de la mano de obra. Factores como } \\
\text { el estado del tiempo, la temperatura, las condiciones del suelo o } \\
\text { incluso la cubierta de la zona de trabajo influyen de manera } \\
\text { significativa en los ánimos de los trabajadores. }\end{array}$ \\
\hline Actividad & $\begin{array}{l}\text { Este factor se refiere a las condiciones que ofrece la obra para realizar } \\
\text { las actividades como; el grado de dificultad, el riesgo al que están } \\
\text { expuestos los obreros, las interrupciones e interferencias con los } \\
\text { procesos constructivos, el orden de la operación y la tipicidad. }\end{array}$ \\
\hline Equipamiento & $\begin{array}{l}\text { Corresponde a la disponibilidad y confiabilidad de los equipos en el } \\
\text { momento que se requiera para asegurar la efectividad de las acciones } \\
\text { constructivas como: equipos de construcción, un adecuado } \\
\text { mantenimiento, un subministro oportuno y los elementos de } \\
\text { protección personal adecuado para asegurar la integridad física de los } \\
\text { implicados. }\end{array}$ \\
\hline Supervisión & $\begin{array}{l}\text { Hace referencia a la calidad y experiencia de los supervisores para } \\
\text { que el trabajo sea continuo y productivo e intervienen aspectos de; } \\
\text { aceptación de actividades, instrucciones claras, criterios de } \\
\text { seguimiento de la obra, supervisión de actividades y gestión de } \\
\text { calidad de todas las acciones. }\end{array}$ \\
\hline Trabajador & $\begin{array}{l}\text { Se refiere a los aspectos personales que los obreros que influyen de } \\
\text { manera positiva o negativa en el rendimiento de sus obligaciones } \\
\text { laborales y se pueden agrupar por; Situación personal, ritmo de } \\
\text { trabajo, habilidad, conocimientos, desempeño y actitud hacia el } \\
\text { trabajo. }\end{array}$ \\
\hline
\end{tabular}

Fuente: Botero (2002) Autor: Nelson Urgilés (2021)

Todos los factores anteriormente descritos afectan de manera significativa en el comportamiento del personal e influyen de manera positiva o negativa en su rendimiento, es por esta razón que, es necesario implementar criterios de reclutamiento y selección que contemplen estos aspectos para contratar mano de obra califica que contribuya al uso eficiente de los recursos para disminuir costos y aumentar la confiabilidad en los procesos pues, como menciona García \& Tantalean (2012): "la construcción en el contexto de la economía es considerada como una actividad estratégica, tanto por su efecto multiplicador en el dinamismo de otros sectores, como por su capacidad de absorción en el corto plazo de mano de obra no calificada" (p.65). Por tal motivo, lo primordial es 
seleccionar los obreros calificados que cumplan con las características necesarias que demanda el proyecto, especialmente en el sector público.

Por lo antes mencionado, el presente artículo aborda la problemática de la falta de criterio para la selección de mano de obra calificada en el campo de construcción de redes de agua potable y alcantarillado, ya que, según experiencia propia e indagaciones con algunos constructores involucrados en actividades de obra civil, se pudo percatar de la falta de personal calificado para la ejecución de los rubros principales que componen los proyectos, los cuales no cumplen con las destrezas o habilidades necesarias para la realización de ciertas actividades encomendadas, a su vez, esta situación genera problemas de diversas índoles al proyecto, como son: retrasos en los cronogramas, disminución de la calidad de los acabados y perdida de confiabilidad en los procesos constructivos. La situación descrita infiere en pérdidas económicas e influye en el prestigio e imagen de los constructores. Por tales motivos y siendo una problemática actual, el objetivo de la investigación fue determinar técnicas o métodos en donde esta selección de la mano obra sea de manera rápida y accesible, para así obtener el personal calificado y satisfacer las necesidades que el proyecto requiere en su fase de ejecución para obtener mejores resultados en la entrega de las obras.

\section{Metodologia.}

Esta investigación es de carácter cualitativo puesto que está orientada al estudio de la realidad de un fenómeno a través del análisis de datos sobre las destrezas, habilidades y características de los obreros para desarrollar las actividades requeridas en el sector de la construcción civil. Al respecto, los autores Osses, Sánchez, \& Ibáñez (2006) mencionan que en este tipo de investigación se analizan las variables constitutivas de un fenómeno a través de la interacción con los datos aportados por los involucrados de la situación estudiada.

Como primer paso de la investigación, se determinó el universo, población y muestra, en la cual se desarrolló el estudio. El universo seleccionado fueron los ingenieros civiles contratistas de la ciudad de Cuenca, de los cuales se eligió la muestra perteneciente a los ingenieros con experiencia en proyectos de construcción de redes de agua potable y alcantarillado en la ciudad en los últimos 5 años, es por esta razón, que se prefirió a los profesionales constructores dentro de la base de datos de la Empresa ETAPA EP, la misma que es la encargada del saneamiento de la ciudad de Cuenca, la cual incluyen este tipo de obras, objeto de este estudio.

En segundo lugar, se realizó una operacionalización para identificar las variables más representativas de las aptitudes de la mano de obra considerada como calificada para la ejecución de proyectos de construcción de redes de agua potable y alcantarillado en la ciudad de Cuenca. De lo mencionado, se determinó que los indicadores de las aptitudes mencionadas se rigen en 4 dimensiones que son:

- Características generales; edad, salud, resistencia, educación, cooperación, y destreza 
- Características laborales; Seguridad social, disponibilidad de EPP, Experiencia, Tipo de contrato

- Características técnicas; Conocimiento en el área, manejo de instrumentos específicos, cumplimiento de tareas

Una vez que se identificaron las dimensiones e indicadores, se procedió a elaborar un instrumento de recolección de datos, que fue aplicado a la población en la base de datos de la Empresa ETAPA EP. Para dar una mayor relevancia y pertinencia a los datos recolectados, se determinó la muestra a través de la fórmula 1.

Fórmula 1. Cálculo del tamaño de muestra (número de encuestas a realizar)

$$
n=\frac{k^{\wedge} 2 * p^{*} q^{*} N}{\left(e^{\wedge} 2 *(N-1)\right)+k^{\wedge} 2 * p^{*} q}
$$

Donde:

- $\quad \mathrm{N}$ : Es el tamaño de la población o universo (número total de posibles encuestados).

- $\quad \mathrm{k}$; Es una constante que depende del nivel de confianza que asignemos. El nivel de confianza indica la probabilidad de que los resultados de nuestra investigación sean ciertos: un 95,5\% de confianza es lo mismo que decir que nos podemos equivocar con una probabilidad del $4,5 \%$.

- e; Es el error maestral deseado. El error maestral es la diferencia que puede haber entre el resultado que obtenemos preguntando a una muestra de la población y el que obtendríamos si preguntáramos al total de ella

- p; es la proporción de individuos que poseen en la población la característica de estudio. Este dato es generalmente desconocido y se suele suponer que $\mathrm{p}=\mathrm{q}=0.5$ que es la opción más segura.

- q; es la proporción de individuos que no poseen esa característica, es decir, es $1-p$

- $\mathrm{n}$; es el tamaño de la muestra (número de encuestas que se va a realizar).

Luego de identificar y aplicar cada una de las variables en la fórmula, se determinó que el número total de encuestas a realizar es de 65 con un porcentaje de confianza del $95.5 \%$. Las preguntas de la encuesta se realizaron en base a una escala Likert, es decir de opción múltiple con 5 posibles respuestas desde "No es importante" hasta "Muy importante". Las preguntas se muestran a continuación en la tabla 2.

Tabla 2. Preguntas de la encuesta

No.

\section{Pregunta}

¿Qué rango de edad considera que deberían tener los obreros para seleccionar la

1 mano de obra calificada dentro de los proyectos de construcción de redes de agua potable y alcantarillado? 
2 ¿Qué rango de importancia considera Usted respecto al estado de salud de los obreros al momento de seleccionar la mano de obra calificada?

¿Qué rango de años de experiencia estima Usted que deberían tener los obreros

3 para considerarse como mano de obra calificada dentro de los proyectos de construcción de redes de agua potable y alcantarillado?

¿Qué tipo de contextura física estima Usted que deberían tener los obreros para considerarse como mano de obra calificada dentro de los proyectos de construcción de redes de agua potable y alcantarillado?

¿Qué nivel de estudios considera Usted que deberían tener los obreros para

5 seleccionar la mano de obra calificada dentro de los proyectos de construcción de redes de agua potable y alcantarillado?

¿Cuántas cargas familiares considera importante que deberían tener los obreros para obtener los perfiles adecuados al momento de elegir la mano de obra calificada?

¿En los proyectos de construcción de redes de agua potable y alcantarillado que

7 número de horas extras consideraría Usted que deben estar dispuestos a realizar los obreros?

¿Considera Usted importante que el obrero conozca sus derechos y obligaciones en lo referente a la seguridad social al momento de seleccionar la mano de obra calificada?

¿Cuáles son los conocimientos empíricos importantes que considera Usted que el obrero debe conocer al momento de seleccionar la mano de obra calificada dentro de los proyectos de construcción de redes de agua potable y alcantarillado?

¿Qué porcentaje de conocimientos considera Usted que el obrero deba conocer en lo referente a las actividades descritas dentro de los proyectos de construcción de redes de agua potable y alcantarillado?

Con el análisis de los resultados de estos datos, se elaboró una plantilla con criterios de selección de los obreros, para implementar en las entrevistas con el fin del calificar las aptitudes de los postulantes de las obras civiles, para realizar una selección de la cuadrilla más óptima.

\section{Resultados.}

Para la presentación de los resultados de la encuesta, se realizó un análisis por pregunta, para identificar cuáles son los factores o aptitudes que, a criterio de los encuestados influye de manera significativa para considerar a la mano de obra como calificada para los proyectos civiles enfocados a redes de agua potable y alcantarillado en la ciudad de Cuenca. El primer análisis de las respuestas, mostrado en la figura 1, corresponde al rango de edad adecuado de la mano de obra. 
Figura 1. Respuestas de la pregunta 1 de la encuesta.

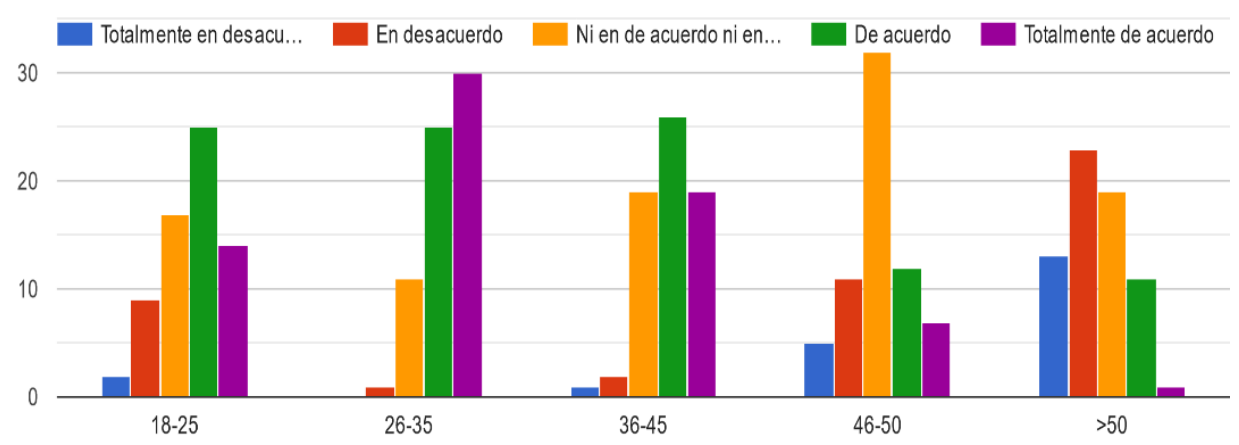

En el grafico anterior se puede apreciar que existe una preferencia entre los obreros con rango de edad entre los 18 y 45 años, sin embargo, se puede notar una predilección por la mano de obra que se encuentra entre los 26 y 35 años. El siguiente criterio, mostrado en la figura 2 de la encuesta, corresponde al grado de importancia que concede la muestra al estado de salud y la calidad de la mano de obra al momento de la selección.

Figura 2. Respuestas de la pregunta 2 de la encuesta.

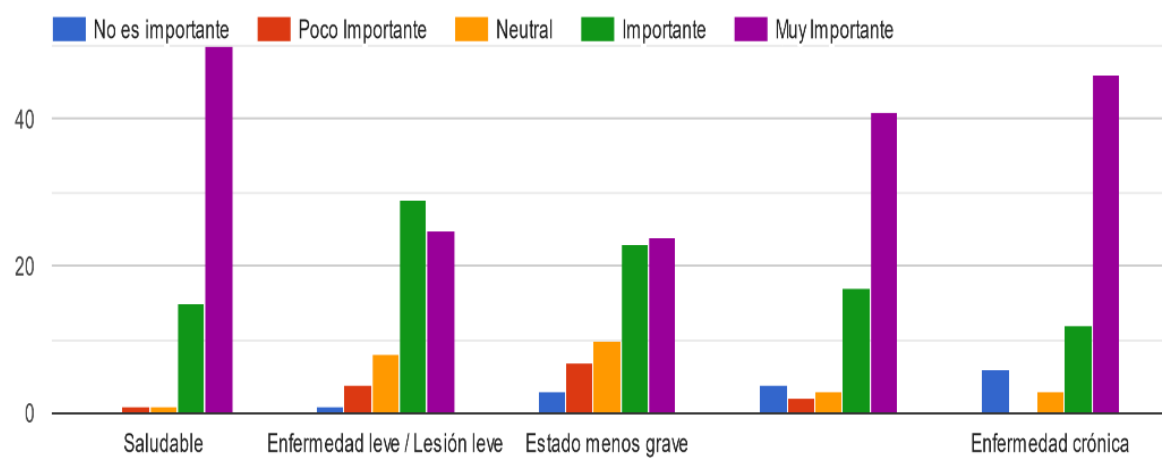

Como se aprecia en la figura anterior, la calidad de la mano de obra está condicionado por su estado de salud, que, para este caso, se considera a las personas saludables como un obrero de calidad, mientras que personas que padezcan enfermedades grabes o enfermedades crónicas, no podrán realizar las actividades inherentes a las construcciones civiles. A continuación, se analiza en la figura 3 la importancia entre los años de experiencia de la mano de obra y la calidad.

Figura 3. Respuestas a la pregunta 3 de la encuesta.

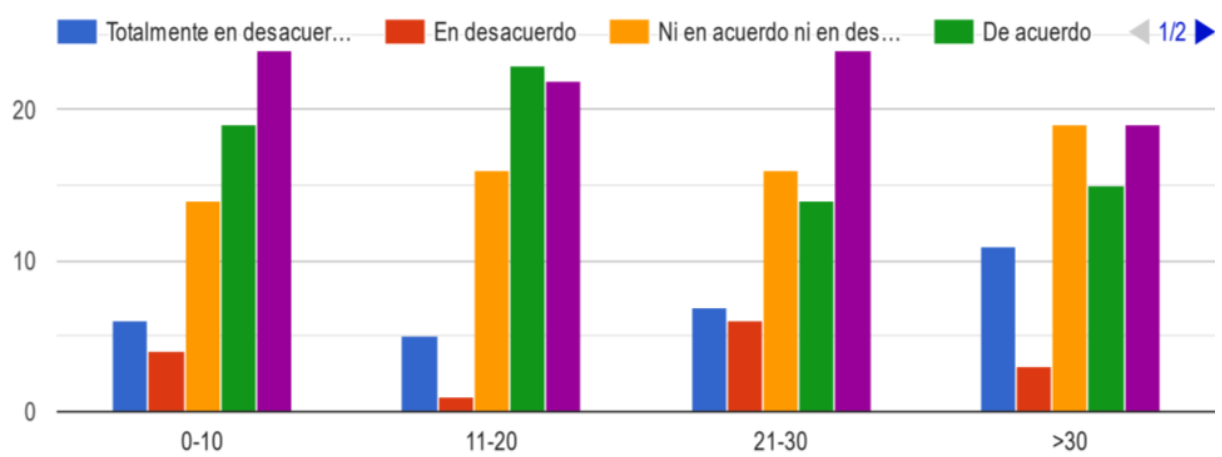


En esta figura se puede apreciar que los años de experiencia no es un factor determinante para las obras civiles pues, como revelan las respuestas de los encuestados la calidad de la mano de obra es independiente a su experiencia, a excepción de aquellas personas que tienen más de 30 años de experiencia por consideraciones de edad, pues, quienes cumplan con más de 30 años de experiencia debe poseer un estimado de 48 años de edad y como se observó en la figura 1, este es un rango de edad considerado como no óptimo para las obras civiles. El siguiente factor, considerado en la figura 4, es la relación entre la contextura física de la mano de obra y la calidad de sus servicios.

Figura 4. Respuestas de la pregunta 4 de la encuesta.

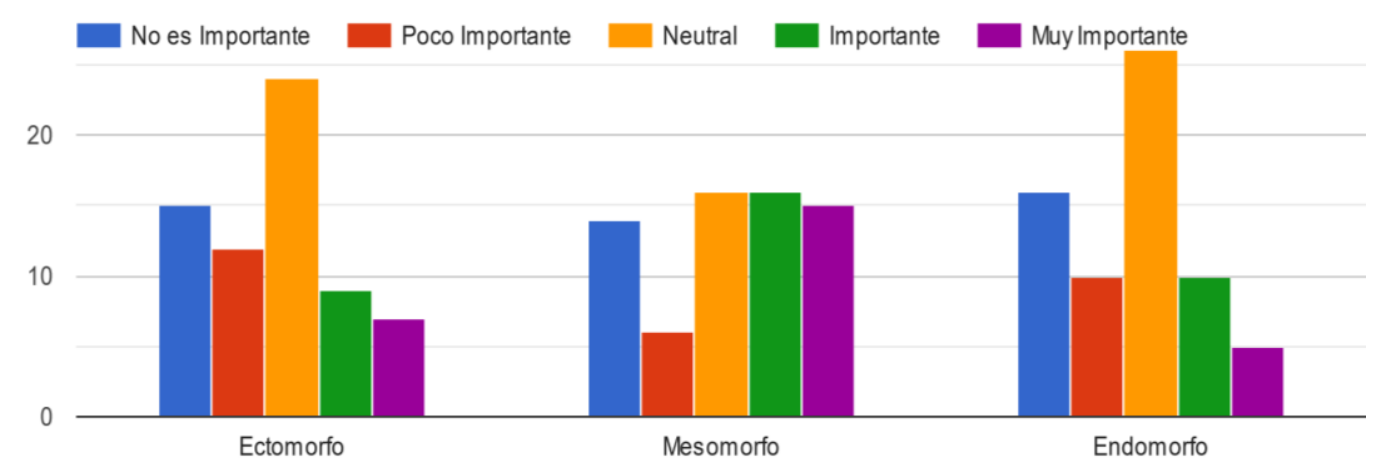

Como se puede observar en la figura 4, la contextura no es un factor determinante para la selección de mano de obra calificada para las obras civiles, pese a esto se puede observar una preferencia parcial por la contextura mesomorfa. El siguiente criterio, mostrado en la figura 5, fue la influencia de la instrucción del trabajador y su calidad en las obras civiles.

Figura 5. Respuestas de la pregunta 5 de la encuesta.

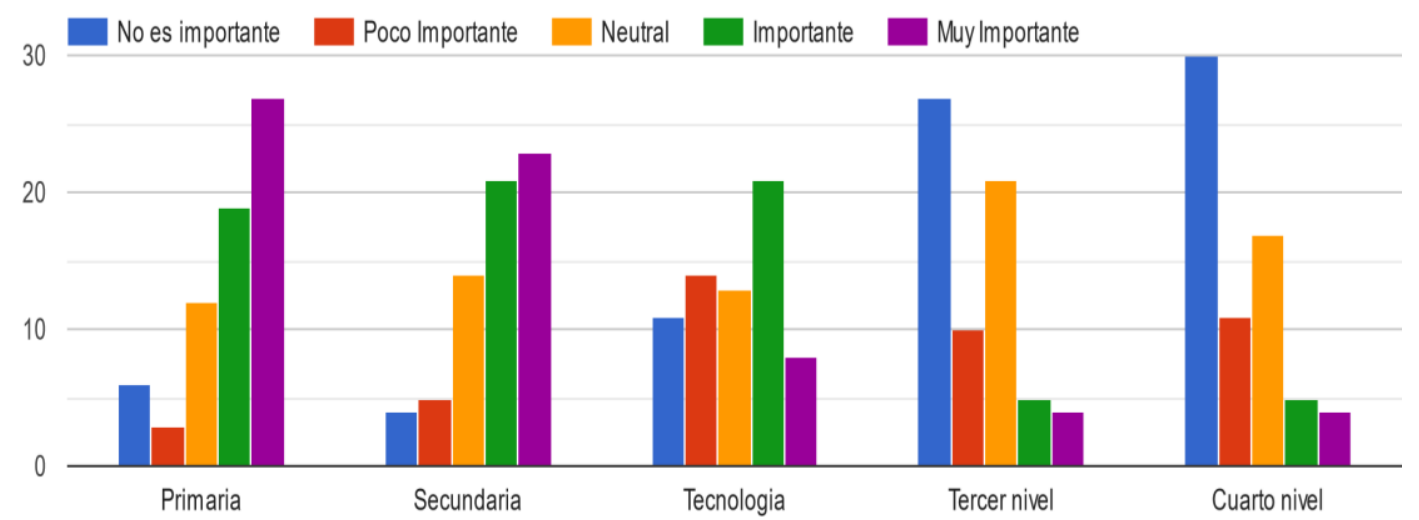

Como se pudo observar, la calidad de la mano de obra no está condicionada al nivel superior de educación por lo cual, se prefiere personas con niveles de instrucción primaria o secundaria y en menor medida a aquellas personas que posean con un título tecnológico. Para el siguiente criterio, mostrado en la figura 6, se analiza la relación entre las cargas familiares y la calidad de la mano de obra. 
Figura 6. Respuestas de la pregunta 6 de la encuesta.

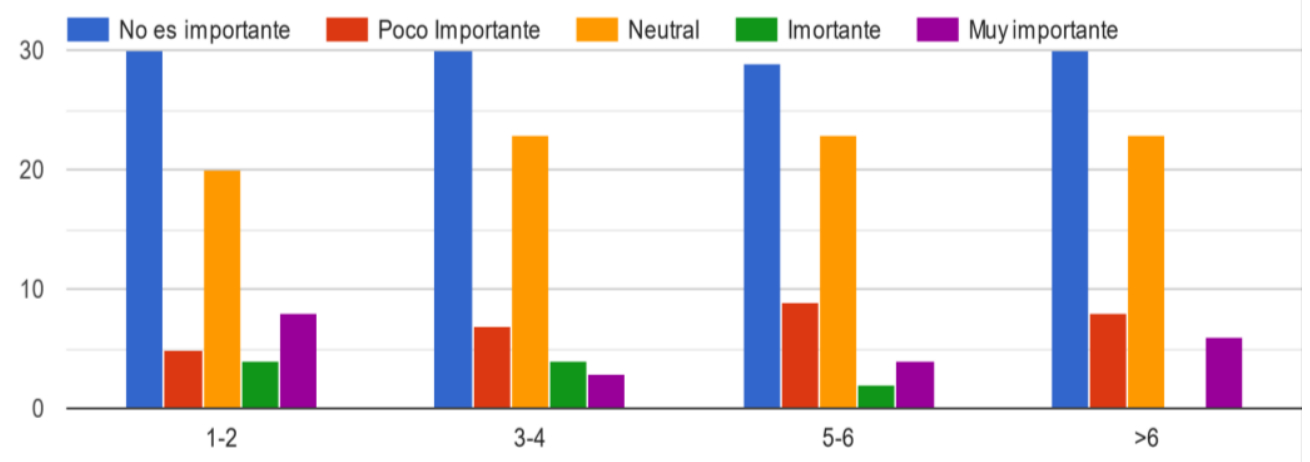

Como se denota en la figura, las cargas familiares no representan un factor determinante en la calidad de la mano de obra en proyectos civiles. El siguiente criterio para analizar en la encuesta es la disposición del personal por trabajar horas extras, lo que se presenta en la figura 7.

Figura 7. Respuestas de la pregunta 7 de la encuesta.

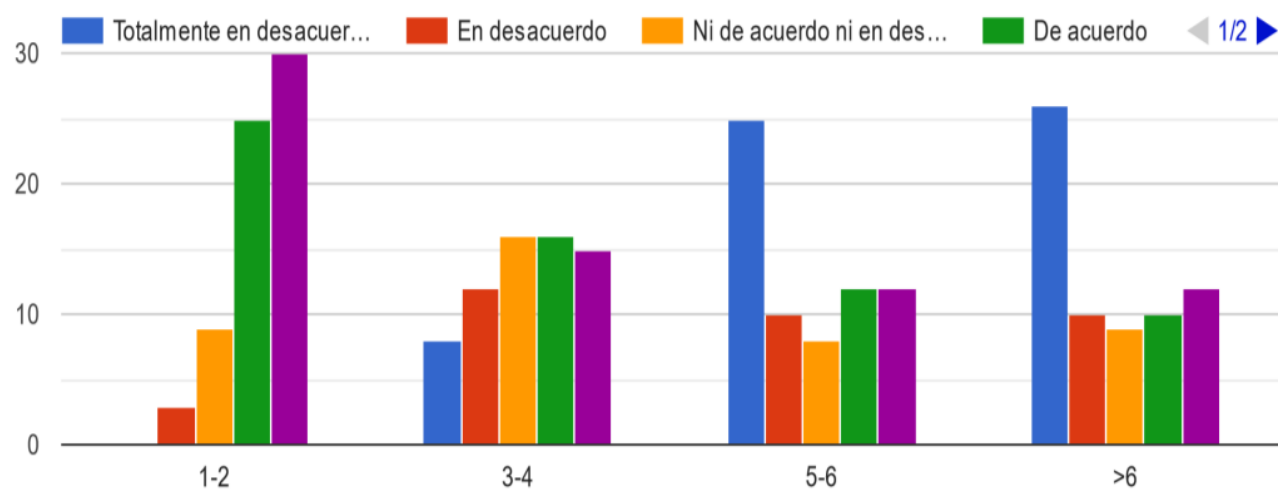

Como se puede evidenciar, la predisposición del obrero para trabajar por lo menos entre 1 a 2 horas extras en su jornada laboral es considerado un factor importante para determinar la calidad de la mano de obra en las construcciones civiles. Como siguiente factor, se muestra en la figura 8, la relación entre el conocimiento del obrero sobre sus derechos y obligaciones respecto a la seguridad social y la percepción de mano de obra calificada de los encuestados.

Figura 8. Respuestas de la pregunta 8 de la encuesta.

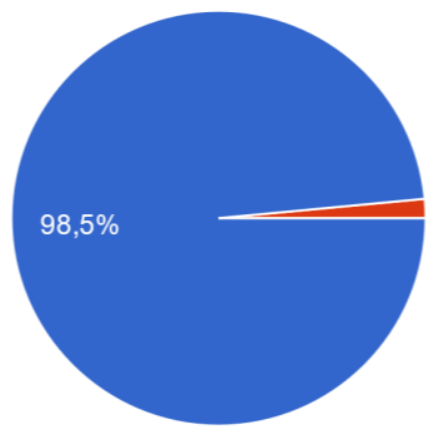


La figura anterior muestra que los encuestados están de acuerdo con una amplia mayoría que, la mano de obra calificada debe conocer sus derechos y obligaciones con la seguridad social. A lo expuesto, el siguiente factor considerado, presentado en la figura 9, hace referencia al tipo de conocimientos empíricos que deben poseer los aspirantes.

Figura 9. Respuestas de la pregunta 9 de la encuesta.

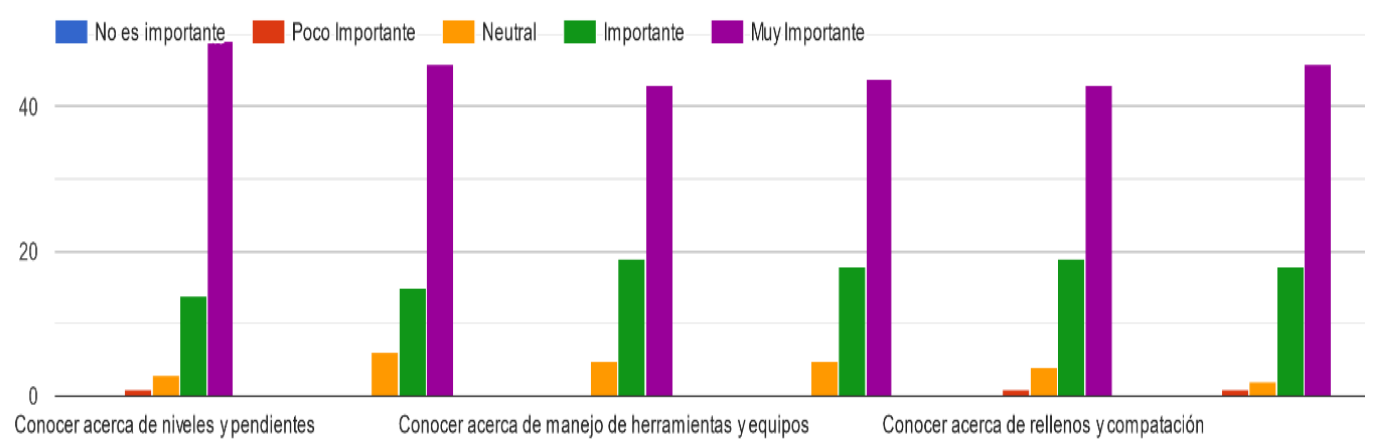

Se puede apreciar en las figuras anteriores que no existe una tendencia clara de preferencia entre los diferentes conocimientos de la mano de obra, pues, según los datos recabados tienen un orden parecido de importancia, por lo que se puede argumentar que la mano de obra de calificada debe tener un conocimiento empírico ampliado para ser considerado como mano de obra calificada, o realizar la selección en base a las funciones que va a realizar el obrero.

Con los datos anteriormente analizados, se procedió a la elaboración de una plantilla, con criterios de selección para la mano de obra calificada en proyectos civiles, enfocados a alcantarillado y redes de agua potable en la ciudad de Cuenca. (ver tabla 4)

Tabla 4. Criterios de selección de acuerdo a las respuestas de la encuesta.

\begin{tabular}{|c|c|c|}
\hline Criterio & Preferencia & Condición idónea \\
\hline Edad & $\begin{array}{c}\text { Obreros con edades } \\
\text { comprendidas entre } 18 \text { y } 45 \\
\text { años }\end{array}$ & $\begin{array}{l}\text { El rango adecuado es entre } 26 \text { y } \\
35 \text { años de edad }\end{array}$ \\
\hline Salud & $\begin{array}{l}\text { Mano de obra saludable o con } \\
\text { lesiones leves que no afecte su } \\
\text { productividad }\end{array}$ & $\begin{array}{c}\text { La condición adecuada es } \\
\text { saludable }\end{array}$ \\
\hline $\begin{array}{l}\text { Experiencia } \\
\text { laboral }\end{array}$ & $\begin{array}{l}\text { Personal con hasta } 30 \text { años de } \\
\text { experiencia }\end{array}$ & El rango adecuado es de 11 a 20 \\
\hline $\begin{array}{l}\text { Contextura } \\
\text { física }\end{array}$ & $\begin{array}{l}\text { Ectomorfo, mesomorfo o } \\
\text { endomorfo }\end{array}$ & Mesoformo \\
\hline Formación & Primaria o secundaria & Primaria \\
\hline $\begin{array}{l}\text { Cargas } \\
\text { familiares }\end{array}$ & Indiferente & Indiferente \\
\hline $\begin{array}{l}\text { Predisposición } \\
\text { a horas extra }\end{array}$ & De uno a seis horas extras & De uno a dos horas extras \\
\hline
\end{tabular}




\begin{tabular}{ccc}
\hline $\begin{array}{c}\text { Conocimiento } \\
\text { sobre } \\
\text { seguridad } \\
\text { social }\end{array}$ & $\begin{array}{c}\text { Conocimiento sobre } \\
\text { responsabilidades y obligación } \\
\text { de la seguridad social }\end{array}$ & $\begin{array}{c}\text { Conocimiento sobre } \\
\text { responsabilidades y obligación de } \\
\text { la seguridad social }\end{array}$ \\
$\begin{array}{c}\text { Niveles, entibados, manejo de } \\
\text { herramientas, mediciones, } \\
\text { empíricos }\end{array}$ & $\begin{array}{c}\text { rellenos, colación de tuberías, } \\
\text { plomería, hormigones, } \\
\text { encofrados. }\end{array}$ & $\begin{array}{c}\text { Conocimiento concreto sobre } \\
\text { niveles, herramientas, mediciones, } \\
\text { rellenos y colocación de tuberías. }\end{array}$ \\
\hline
\end{tabular}

Los criterios de selección de mano de obra calificada presentados en la tabla anterior, son un conjunto de consideraciones realizadas por Botero sobre los factores que afectan la productividad de los obreros y la percepción de los ingenieros civiles que trabajan en obras civiles de alcantarillado y redes de agua potable. En este contexto, se realizó una diferenciación entre las preferencias que conciernen a un conjunto globalizado de características permisibles a la contratación de personal y las condiciones idóneas o ideales para el trabajo, con la finalidad de proporcionar a los contratistas una visión mucho más amplia sobre las características que debe poseer su cuadrilla de trabajo.

\section{Conclusiones.}

- Se determinaron los factores que influyen en la selección de la mano de obra calificada en las obras civiles, a través de un análisis bibliográfico, y se verificó que existen varias características que la gestión debe considerar para consumir los recursos humanos de manera adecuada, como: la actividad, las características propias del trabajador, condiciones laborales, entre otros.

- La información recopilada de la encuesta demostró que, para que la mano de obra sea considerada como calificada en los proyectos civiles debe cumplir con algunos criterios. El primer criterio hace referencia a las características propias del trabajador como la edad, salud, experiencia laboral, contextura y formación. Pero, pese a que la mayoría de las respuestas a estos cuestionamientos corresponden a un perfil "normal" de un trabajador como es el hecho de que posea buena salud, que sea joven, y que posea experiencia en el campo laboral, el análisis de los datos determinó aspectos interesantes como el hecho que la condición física, contrario a lo que se puede pensar, no es determinante para clasificar a una persona como mano de obra calificada. Por otro lado, un nivel de formación superior al primario tampoco representa un impedimento para considerar a una persona como un recurso humano de calidad.

- Finalmente, el análisis de datos demostró que, pese a que las condiciones personales de los obreros influyen en su clasificación como de mano de obra calificada, los conocimientos empíricos como el manejo de herramientas, mediciones, habilidades de construcción y predisposición por trabajar horas extras, tienen la misma relevancia para la selección de 
personal enfocado a la construcción de obras civiles de agua potable y alcantarillado.

- Agradecimientos: El presente artículo es parte del trabajo de investigación y titulación del Programa de Maestría en Construcción con Mención en Administración de la Construcción Sustentable de la Universidad Católica de Cuenca, por ello agradezco a todos y cada uno de los instructores por los conocimientos e información brindados para la elaboración del trabajo.

\section{Referencias bibliográficas.}

Botero, L. (2002). Análisis de rendimientos y consumos de mano de obra en actividades de construcción. Revista Universidad EAFIT. (128), 9-23. https://n9.cl/9m1wn

Cruz, N., \& Centeno, E. (2019). La construcción epistemológica en Ingeniería Civil: Visión de la Universidad de Costa Rica. UCR, 19(1), 1-30. doi:10.15517/aie.v19i1.35328

García, T., \& Tantalean, I. (2012). Selección y control del factor humano en empresas de construcción civil. Producción y gestión, 15(2), 63-73. https://n9.cl/bulng

Giordani, C., \& Leone, D. (2015). Ingeniería Civil. Departamento de Ingeniería civil de la UNT. https://n9.cl/1yi6c

Mejía, G., \& Hernández, T. (2007). Seguimiento de la productividad en obra: Técnicas de medición de rendimiento de la mano de obra. UIS Ingenierías, 6(2), 45-59. https://n9.cl/byh23

Mundo Constructor. (5 de abril de 2021). Gestión de la calidad en los procesos constructivos en la situación actual. https://n9.c1/5otlj

Osses, S., Sánchez, I., \& Ibáñez, F. (2006). Investigación cualitativa en educación. hacia la generación de teoría a través del proceso analítico. Estudios Pedagógicos, 32(1). doi:http://dx.doi.org/10.4067/S0718-07052006000100007

Padilla, A. (2016). Productividad y rendimiento de mano de obra para algunos procesos constructivos seleccionados en la ejecución del edificio ISLHA del ITCR. tesis de grado, Instituto tecnológico de Costa Rica. https://n9.cl/sraj

Salanova, M., Gracia, E., \& Lorente, L. (2007). Riesgos psicosociales en trabajadores de la construcción. Gestión practica de riesgos laborales, 44, 1-8. https://n9.cl/35wj

Universidad de Chile [CEC]. (2003). Métodos Constructivos; catedra CI52A. Repositorio de la CEC. https://n9.cl/n8xjr

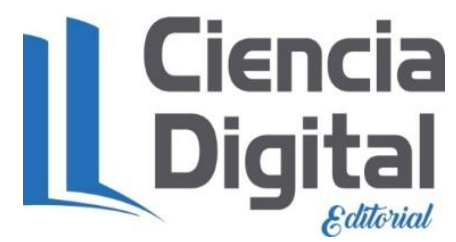




\section{PARA CITAR EL ARTÍCULO INDEXADO.}

Urgilés Martínez, N. S., \& Ortega-Castro, J.-C. (2021). Criterios de selección de mano de obra calificada para un proyecto de construcción de redes de alcantarillado y agua potable en la ciudad de Cuenca. ConcienciaDigital, 4(3), 40-53. https://doi.org/10.33262/concienciadigital.v4i3.1763

\section{¿Ciencia}

El artículo que se publica es de exclusiva responsabilidad de los autores y no necesariamente reflejan el pensamiento de la Revista Conciencia Digital.

El artículo queda en propiedad de la revista y, por tanto, su publicación parcial y/o total en otro medio tiene que ser autorizado por el director de la Revista Conciencia Digital.

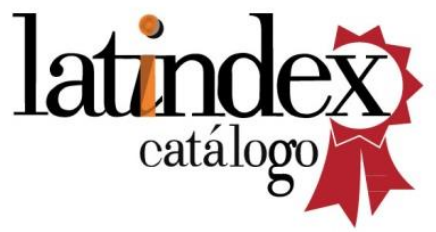

